Acantholysis 391

Acanthosis, nigricans 164

Acitretin 3

Acne 218, 278

-, closed comedonal 278

- inversa 261

- vulgaris 273

Adapalene 17, 218

Adverse effects 288

- events 375

Aftersun lotion 52

Albright's hereditary osteodystrophy 209

Alcohol 437

- dehydrogenase 93

Alopecia areata 415,418

Amylase 305

Anetoderma, familial 321

Angiomyxoma 195

Angiopathy 314

Antifungal 382

Antioxidant capacity 336

Antioxidative parameter, total radical 336

Aphthosis, severe 247

Apoptosis 5

Arthritis, acute 406

Ascorbic acid 52, 336

Aspartic protease 412

- _ inhibitors 412

Atopic dermatitis 56, 98, 145, 153, 321

Autoantigen 330

Autografts, cultured epithelial 101

Autoimmune bullous diseases 118 , 305

Autosomal dominant trait 107

Baldness, male pattern 231

Basement membrane zone 330

Behçet's disease 33, 243, 247

Benzoyl peroxyde 273

Bioavailability, topical 44

Bioengineering 351

Bioterrorism 108

Birthmark 327

Blistering, subepidermal 310

Blood ionized calcium 90

Bone 73

Bullous disease 330

Buschke-Ollendorf syndrome 307

Calcinosis cutis 209

- -, dystrophic 90, 174

-, idiopathic cutis of the penis 174

Calciphylaxis 423

Calcipotriol 375

Cancer 187, 321

Candida albicans 412

Capillaritis 175
Carcinoma 187

-, squamous cell 192, 206

Chemokines 118

Chemotherapy 73, 288

Childhood 284

Chromosome Xp21 region deletion 98

Chronic graft-versus-host disease 370

- inflammation 336

- urticaria 130

Classification 426

Clinical heterogeneity 167

- severity 167

Clubbing 173

Collagen metabolism 385

Colorimetry 218

Comedolysis 218

Common variable immunodeficiency 156

Compression therapy 439

Congenital valvular aplasia 101

Consensus report 145

Contact dermatitis 106

Corticosteroid 11, 305

Cost-effectiveness 404, 405

Cryobiology 111

Cryoglobulinemia 26

Cryosurgery 111

Cryotherapy 111,162

Cutis laxa, acquired 346

Cyclosporin A 145, 198, 320, 321 . 415

Cyst, epidermal 174

Darier's disease 167

Dendrocyte 184

Dermatofibrosarcoma protuberans 37

Dermatoma 105

Dermatomycosis 382

Dermis 317

Diabetes mellitus 177

- -, insulin-dependent 314

Dietary therapy 340

Docetaxel 288

Doppler flow 355

Dowling-Degos disease 176

Downut sign 180

Dyshidrotic eczema 265

Eczema, hand 351

Efficacy 56

Ehlers-Danlos syndrome 385

Elastase I 305

Elastases 346

Elastic nevus 307

Elastin metabolism 307

Enalapril 391

Enkephalin 11
Eosinophilic pustular folliculitis 86

Epidermolysis bullosa acquisita 310

Epiluminescence light microscopy 420

Equipment 111

Eradication therapy 130

Erythema 256

-, elevatum diutinum 79

- multiforme, herpes-simplexvirus-associated 234

Erythroderma 65

Extracorporeal photophoresis 140

Factor XIIIa 184

Fasciitis 431

Fatty aldehyde dehydrogenase 93

Fibromatosis, juvenile hyaline 18

Fibroxanthoma, atypical 29, 37

Fixed drug eruption, multiple 291

5-Fluorouracil, intravenous 394

Fractal quantification 212

FTA-Abs test 362

Glomangioma 270

Glomus tumors 270

Glucocorticosteroid 61

Glycation 177

Glycerol kinase defiency 98

Graft, keratinocyte 101

-, punch 133

- , test 133

Graft-versus-host reaction 184

Granulocyte colony-stimulating factor 301

Granuloma 156, 317, 434

-, extrafacial 79

- , faciale 79

Grover's disease 410

Growth factor 301

Halogenoderma 295

HBV vaccination 1

Helicobacter pylori 130

Hemolytic-uremic syndrome 179

Hepatitis C virus infection 26

Herpes-simplex-virus-associated, erythema multiforme 234

Histamine, plasma level 252

Histamine-releasing agents 426

Histiocytic disorder 434

Histiocytoma, malignant fibrous 29 , 37

History 111

HIV 126, 256, 362

- infection 362

HPV 122, 206

HSV 234

Hyalin 18,83

Hyalinosis, infantile systemic 18
Hyalonisis, delayed-onset 83

-, systemic 83

Hyperandrogenism 278

Hyperelasticity 385

Hyperparathyroidism 423

Hypersensitivity syndrome 388

-, delayed-type 291

Hypopigmentation 327

Hypothyroidism 164

Ichthyosis 93

-, X-linked recessive 231

Image analysis 355

Immunocompetence 73

Immunocompromised patient 162

Immunoglobulins 156

Immunoglobulin A 330

Immunohistochemistry 118

Immunosuppression 29, 317, 415

Impetigo herpetiformis 61,400

In vitro fertilization 107

Inhibition spectrum 252

Insect bite 159

Insulin resistance 164

Interferon $\alpha 103,418$

Iododerma 295

Isotretinoin 278, 406

-, oral 404, 405

Itraconazole 100,382

Kaposi pseudo-sarcoma 439

- sarcoma 73,420

Keratinization 167

Keratoacanthoma 122

-, familial 394

-, multiple familial 394

Keratosis follicularis 167

Lactulose permeability test 153

Laser 314

- therapy 355

Leg ulcer 101, 423

Leiomyosarcoma 37

Leopard syndrome 385

Leucocytoclasic vasculitis 26

Lichen planus 1, 301

Lichenoid drug reaction 301

Linuche unguiculata 171

Lipids 340

Lipomatosis, benign symmetrical 437

Lipoproteins 243

Liposomes, phospholipid 273

Livedo 423

Liver cirrhosis 437

Loss of heterozygosity 270

Low-salt water 153

Lung cancer 173

Lupus erythematosus, subacute cutaneous 90
KARGER

(C) 1998 S. Karger AG, Basel

Fax + 41613061234

E-Mail karger@karger.ch

www. karger.com
Accessible online at:

http://BioMedNet.com/karger 
Lymphangitis, nodular 159

Lymphoma, cutaneous 284

-, malignant 75,100

Magnetic resonance 73

Management 426

Mastocytosis 426

Medusa stage 171

Melanocytes 5

Melanocytosis, dermal 105

Melanoma, cutaneous 212

-, malignant 187

Metal workers 351

Metastasis 298

Methotrexate 177

Methoxypsoralen 140

Micrographic surgery 37

Microsporum canis 317

Microvasculature 212

Mohs, slow 37

-, surgery 37

Monoclonal gammopathy 434

Mosaicism 270

Mycobacterial lymphadenitis 97

Mycobacterium terrae 97

Mycosis follicularis 284

- fungoides 284

$\mathrm{N}$-acetyl-5-methoxytryptamine 52

Naevus, anemicus 327

-, congenital 327

-, pharmacological 327

Nail 206, 288

Necrobiotic granuloma 394

Neoplasm 173

Neuropathy 314

Nitric oxide 33

Nitroglycerin 106

Nocardiosis, lymphocutaneous 159

Nodular fasciitis 431

Non-Hodgkin's lymphoma 75

Nonimmunocompromised host 97

Nonionic contrast media 291

Nose 192

\section{Obesity 164}

Ofuji's disease 86

Oncocytoma 322

Onychomycosis 382

Oocyte donation 107
Orf 162

Osteoma cutis 209

Osteomyelitis 314

Oxidation 243

Oxidative stress 52

Pachyonychia congenita 107

Pancreatic carcinoma 182

Pancreatitis 305

-, corticosteroid-induced 305

Panniculitis 182

Paraneoplastic syndrome 173

Paraproteinemia 203

Pathogenicity 412

Pemphigoid, bullous 140, 310, 322

-, cicatricial 310

Pemphigus 391

- foliaceus 140

- vulgaris 140

Percutaneous absorption 44

Permabond technique 273

Photophoresis, extracorporeal 370

Photosensitivity 388

Phototherapy 375

Pigmentation 256

Plague 108

Plasma cells 434

Pleomorphism 69

Poisoning 44

Polyneuropathy 423

Pompholyx 265

Porocarcinoma 298

Port wine stains 355

Pregnancy 3, 61, 400

Prognosis 278

Proliferating cell nuclear antigen 431

Propionibacteria 273

Pseudoparathyroidism 209

Pseudotumor 431

Psoriasis $11,65,223,320,321,375$

-, eyelid 223

-, generalized pustular 61

-, malignancy 320

-, mentally ill patients 229

-, scalp 226

-, skin folds 225

Puerperium 400

PUVA 203

Pyodermia fistulans sinifica 261
Radar 403

Radio frequencies 403

Reactive oxygen species 336

Recombinant proteins 412

Recurent neoplasia 195

Relapse 278

Remission 167

Renal transplant recipient 29

Resistance 278

Reticulate pigmented anomaly of flexures 176

Retinoid-PUVA treatment 61

Retinoids 3

Retrovirus 86

Risk groups 126

Safety 382

Salicylic acid 44

Sandimmun 56

-, Neoral 56

Sarcoma, cutaneous 29

Schamberg's disease, familial 175

Scleroedema 177

- diabeticorum 177

Scleromyxoedema 180

Sclerosis, systemic 75

Sclerotic fibroma 69

Seabather's eruption 171

Seborrheic dermatitis 126

Segmental involvement, type 2 270

Severity index 265

Side effects, cutaneous 418

Sjögren-Larsson syndrome 93, 340

Skin 187,403

- barrier function 98

- charges 437

- diseases 11

- tumor 122

Smoking adverse effects 261

Soft tissue tumor 195

Sporotrichin test 100

Sporotrichosis 100

Squamometry 218

Steroid sulfatase deficiency 231

Subcorneal pustular dermatosis 203

Sulfasalazopryridine 388

Syphilis 362
Tape stripping 44

T-cell, human lymphotrophic virus 186

- leukemia lymphoma, adult 103

- lymphoma, cutaneous 179

- repertoire 234

TCR 234

Techniques 111

Teratogenesis 3

Teratogenicity 404, 405

Therapy 298

$\alpha$-Tocopherol 52

Tolerability 56, 382

Topical administration 273

- therapy 230

Toxic epidermal necrolysis 184,198

Transdermal therapeutic system 106

Transient acantholytic dermatosis 410

Tretinoin 218

Trypsin 305

Tumor, inflammatory myofibroblastic 431

-, malignant peripheral 37

Ultraviolet light 256

Urate 336

Urticaria 346

-, solar 252

UV irradiation 5

Vasculitis 79

VDRL test 362

Vegetating iododerma 295

Venous insufficiency, chronic 439

Vindesine 73

Viral gene expression 234

Vitamin D 11

Vitiligo 65,133

Vogt-Koyanagi-Harada disease 65

Wound healing 184,314

Xanthoma 434

Yersin, Alexandre 108

Zidovudine 103 